\title{
On a definition of seminorm in $\mathbf{W}^{s, p}(\Gamma)$
}

\author{
FABIO Gastaldi and GianNi GILARDI
}

\begin{abstract}
A definition of seminorm is given in the Sobolev space $\mathbf{W}^{s, p}(\Gamma)$ on a smooth compact manifold $\Gamma$ without boundary, using a localization procedure without partition of unity.
\end{abstract}

\section{INTRODUCTION}

In the study of noncoercive boundary value problems for elliptic partial differential equations or inequalities, one of ten can estimate derivatives (or, more generally, difference quotients) of the solution, but not the solution itself.

On the other hand, in the linear case, estimates of high order norms of the solution (for instance, in Sobolev spaces) are well known, depending on suitable norms of data and on lower order norms of the solution itself. Similar estimates can be obtained also in several non linear problems; they can have either a local character or a global one. Yet, local a priori estimates of high order norms of the variation of the solution are often useful, involving only lower order norms of the same variation. In such a case, partitions of unity are not useful, since they generally call into play norms of zero order, for instance the $\mathbf{L}^{p}$ norm of the solution, which is not controlled in noncoercive problems, usually.

Thus, it could be useful to introduce functional seminorms whose definition is given in terms of local charts, without using partition of unity. In this paper we develop such an idea in the framework of the Sobolev space

Partial support for this work was provided by lstituto di Analisi Numerica del C.N.R. di Pavia. 1980 Mathematics Subject Classification (1985 revision): $46 \mathrm{E} 35$.

Editorial de la Universidad Complutense. Madrid, 1989. 
$\mathbf{W}^{s, p}(\Gamma)$, with $s>0,1<p<\infty, \Gamma$ being a compact smooth manifold without boundary.

An application of this technique is given in [GG].

\section{SETTING OF THE PROBLEM}

Throughout this paper $\Gamma$ will denote a manifold in a euclidean space such that

$I^{\prime}$ is $N$-dimensional, compact, without boundary, of class $\mathbf{C}^{m, 1},[1.1]$ where $N \geq 1$ and $m \geq 0$ are integers.

Under this assumption, one can find in the literature several definitions of the Sobolev space $\mathbf{W}^{s \cdot p}(\Gamma)$, for all real $s$ with $0<s \leq m+1$, and of its norm (see $[\mathrm{A}],[\mathrm{BL}],[\mathrm{KJF}],[\mathrm{LM}],[\mathrm{M}],[\mathrm{N}],[\mathrm{T}]$ ).

We are interested here in definitions using localization and local change of variables (see, e.g. [LM] (iii) 2.5; in particular Prop. 2.9): the problem is reduced to a preliminary study of the space $\mathbf{W}^{s, p}(\Omega)$, where $\Omega$ is an open subset of $\mathbf{R}^{N}$. In this paper we introduce a variant to such kind of definitions (see also [N] pp. 88 and 94), which consists in avoiding partitions of unity, in view of a similar definition of a seminorm whose kernel is made by locally constant functions.

In section 3 we will give the definitions of $W(\Gamma)$, of its norm and of its seminorm. Although the definition of the space is clearly independent of the system of local charts, this is no longer true for the norm and for the seminorm; nevertheless, we will show that different systems lead to topologically equivalent norms and even seminorms. This requires a preliminary study of the case where $\Gamma$ is replaced by an open set of $\mathbf{R}^{N}$ : this is done in section 2. Finally, section 4 is devoted to a comparison between our definitions and others, some of which already given in the literature.

Although most of the following could be extended to cover the cases $p=1$ and $p=\infty$, yet we will confine our attention to the case $1<p<\infty$.

\section{THE CASE OF AN OPEN SUBSET OF $\mathbf{R}^{N}$}

We begin by recalling the (usual) definition of $W^{s, p}(\Omega)$ and of its norm and seminorm. 
Definition 2.1 Let $\Omega$ be an open subset of $\mathbf{R}^{N}$. Let $s>0$ and $\left.p \in\right] l,+\infty[$ be given. The space $\mathbf{W}^{s, p}(\Omega)$, its seminorm $|\cdot|_{s, p, \Omega}$ and its norm $\left\|_{\bullet}\right\|_{s, p, \Omega}$ are defined respectively as follows:

(i) if $0<s<1$,

$$
\begin{gathered}
\mathbf{W}^{s, n}(\Omega)=\left\{v \in \mathbf{L}^{p}(\Omega):|v|_{s, p, \Omega}<\infty\right\}, \\
|v|_{s, p, \Omega}^{p}=\int_{\Omega} \int_{\Omega}|x-y|^{-N-s p}|v(x)-v(y)|^{p} d x d y, \\
\|v\|_{s, p, \Omega}=\|v\|_{0, p, \Omega}+|v|_{s, p, \Omega},
\end{gathered}
$$

where $\|\cdot\|_{0, p, \Omega}$ is the norm in $\mathbf{L}^{\prime}(\Omega)$;

(ii) if $s$ a positive integer,

$$
\begin{gathered}
\mathbf{W}^{s, p}(\Omega)=\left\{v \in \mathbf{L}(\Omega): D^{\alpha} v \in \mathbf{L}^{p}(\Omega), \text { for }|\alpha| \leq s\right\}, \\
\|v\|_{s, p, \Omega}=\sum_{|\alpha| \leq s}\left\|D^{\alpha} v\right\|_{0, p, \Omega}, \\
|v|_{s, p, \Omega}=\sum_{j=1}^{N}\left\|\partial_{j} v\right\|_{s-1, p, 1},
\end{gathered}
$$

where $\partial_{j}=\frac{\partial}{\partial x_{j}}, D^{\alpha}=\partial_{1}^{\alpha} \alpha^{\alpha} \partial_{N}^{\alpha}$ and $|\alpha|=\sum_{j} \alpha_{j}$ if $\alpha=\left(\alpha_{1}, \ldots, \alpha_{N}\right)$;

(iii) if $s>1$ and $s$ is not an integer, denoting by $m$ the largest integer $\leq s$, we define

$$
\begin{gathered}
\mathbf{W}^{s, p}(\Omega)=\left\{v \in \mathbf{W}^{m, p}(\Omega): D^{\alpha} v \in \mathbf{W}^{s-m, p}(\Omega), \text { for }|\alpha|=m\right\}, \\
\|v\|_{s, p, \Omega}=\|v\|_{m, p, \Omega}+\sum_{|\alpha|=m}\left|D^{\alpha} v\right|_{s-m, p, \Omega}, \\
|v|_{s, p, \Omega}=\sum_{j=1}^{N}\left\|\partial_{j} v\right\|_{s-1, p, \Omega} .
\end{gathered}
$$

A preliminary step consists in comparing $|\cdot|_{s, p, \Omega}$ with $\sum_{i}|\bullet|_{s, p, \Omega_{i} \text {, where }\left\{\Omega_{i}\right\}}$ is a finite, open covering of $\Omega$, at least when $0<s \leq 1$. To this end we need some lemmas. 
Lemma 2.2 Let $\Omega$ and $\Omega_{i}(i=1, \ldots, n)$ be open, connected subsets of $\mathbf{R}^{N}$ such that $\Omega=\cup \Omega_{i}$. Then at least one among the $\Omega_{i}$ 's is such that the union of the remaining ones is connected.

Proof. By induction, we notice that the result is trivial if $n=2$. Let $m \geq 2$ and assume that the result holds for $n=m$ : we are going to show that it is still valid for $n=m+1$. Let $\Omega$ and $\Omega_{i}(1 \leq i \leq m+1)$ satisfy the assumption: then, $\Omega_{m+1}$ intersects the union of the preceding ones, whence it intersects one of them. We may assume that this is $\Omega_{m}$. Set $\Omega_{i}^{\prime}=\Omega_{i}$ if $1 \leq i<m$ and $\Omega_{m}^{\prime}=\Omega_{m} \cup \Omega_{m+1}$ : by the inductive assumption (which can be applied because $\Omega_{m}^{\prime}$ is connected), one among the $\Omega_{i}^{\prime}$ 's is such that the union of the remaining ones is connected. Let $\Omega_{k}^{\prime}$ be this set. If $k=m$, then $\Omega_{0}=\bigcup_{i<m} \Omega_{i}$ is connected and intersects $\Omega_{m}^{\prime}$, whence it intersects either $\Omega_{m}$ or $\Omega_{m+1}$. Therefore, the set we wanted is either $\Omega_{m+1}$ or $\Omega_{m}$, respectively. If $k<m$, the wanted set is $\Omega_{k}$.

Lemma 2.3 Let $\Omega$ and $\Omega_{i}$ satisfy the same assumptions as in the previous Lemma. Then there exists a permutation $\pi$ of $(1, \ldots, n)$ such that for all $i$, $1<i \leq n$, there exists $j, 1 \leq j<i$, such that $\Omega_{\pi(i)} \cap \Omega_{n(j)} \neq \phi$.

Proof. By induction, we notice that the result is trivial if $n=2$. Let $m \geq 2$ and assume that the result holds for $n=m$ : we are going to show that it is still valid for $n=m+1$. Let $\Omega$ and $\Omega_{i}(1 \leq i \leq m+1)$ satisfy the assumption: by Lemma 2.2, one among the $\Omega_{i}$ 's is such that the union of the remaining ones is connected. Up to a permutation of $1, \ldots, m+1$, we may assume that this is $\Omega_{m+1}$. By the inductive assumption, there exists a permutation $\pi$ of $(1, \ldots, m)$ such that each $\Omega_{\pi(1)}$, apart from $\Omega_{\pi(1)}$, intersects one of the preceding ones: define $\Pi(i)=\pi(i)$ if $1 \leq i \leq m$ and $\Pi(m+1)=m+1$ : $\Pi$ satisfies the requested properties.

In the following we shall use this wel! known result (see [N] Lemma 5.5: the assumption on $\Omega$ can be weakened), which we state as a lemma.

Lemma 2.4 Let $\Omega$ be a bounded open subset of $\mathbf{R}^{N}, m \geq 0$ an integer, $0<s \leq m+1, p \in] 1,+\infty\left[\right.$ and $w \in C^{m .1}(\bar{\Omega})$. Then, for all $v \in \mathbf{W}^{s, p}(\Omega)$ it is $\nu w \in \mathbf{W}^{s, p}(\Omega)$ and

$$
\|v w\|_{s, p, \Omega} \leq c\|v\|_{s, p, \Omega}
$$

where $\mathrm{c}$ does not depend on $v$.

The following lemma is well known: here we prove it under weak assumptions. The following notations will be used. 


$$
B_{r}(x)=\left\{y \in \mathbf{R}^{N}:|x-y|<r\right\} \text { and } B_{r}=B_{r}(0) .
$$

Lemma 2.5 Let $\Omega$ and $\Omega_{i}(i=1, \ldots, n)$ be open bounded subsets of $\mathbf{R}^{N}$ such that $\Omega=\bigcup_{i} \Omega_{i}$, satisfying the following condition:

$$
\forall x \in \bar{\Omega} \exists \epsilon>0, \exists i, 1 \leq i \leq n \text { such that } \Omega \cap B_{\epsilon}(x) \subset \Omega_{i} .
$$

Let $s>0$ and $p \in] 1,+\infty\left[\right.$. Then, all $v \in \mathbf{L}^{p}(\Omega)$ such that $v_{\mid \Omega_{i}} \in \mathbf{W}^{s, p}\left(\Omega_{i}\right)$, $1 \leq i \leq n$, belongs to $\mathbf{W}^{s, p}(\Omega)$; moreover, there is a constant $c>0$ such that, for all $\nu \in \mathbf{W}^{s, p}(\Omega)$,

$$
\|v\|_{s, p, \Omega} \leq c \sum_{i}\left\|v_{\mid \Omega_{i}}\right\|_{s, p, \Omega_{i}}
$$

Proof. Since $\bar{\Omega}$ is compact, there exist $m \in \mathbf{N}, \epsilon_{j}>0$ and $x^{j} \in \bar{\Omega}(1 \leq j \leq m)$ such that, setting $\Omega_{j}^{\prime}=B_{t_{j}}\left(x^{j}\right)$, it is $\cup \Omega_{j}^{\prime} \supset \bar{\Omega}$ and for all $j(1 \leq j \leq m)$ there is $i(1 \leq i \leq n)$ such that $\Omega \cap \Omega_{j}^{\prime} \subset \Omega_{i}{ }^{j}$

Let $\left\{\psi_{j}\right\}$ be a partition of unity associated to the compact set $\bar{\Omega}$ and to the covering $\left\{\Omega_{j}^{\prime}\right\}$. If $v \in \mathbf{L}^{p}(\Omega)$ and $v_{\mid \Omega_{j}} \in \mathbf{W}^{s, p}\left(\Omega_{i}\right), \quad 1 \leq i \leq n$, then $v_{\mid \Omega \Omega_{i} \cap \Omega_{j}^{\prime}} \in \mathbf{W}^{s, p}\left(\Omega_{i} \cap \Omega_{j}^{\prime}\right), \mathrm{I} \leq j \leq m$, and then $\left(\psi_{j} v\right)_{\mid \Omega_{i} \cap \Omega_{j}} \in \mathbf{W}^{s . p}\left(\Omega_{i} \cap \Omega_{j}^{\prime}\right)$. Since supp $\psi_{j}$ has a strictly positive distance from $\Omega / \Omega_{j}^{\prime}$, the direct evaluation of the seminorm leads to $\psi_{j} v \in \mathbf{W}^{s, p}(\Omega), \forall j$. Thus, $v=\sum_{j} \psi_{j} v \in \mathbf{W}^{s, p}(\Omega)$. This evaluation leads also to the following inequality, where we omit the symbol of restriction (which will be done also in what follows):

$$
\left\|\psi_{j} v\right\|_{s, p, \Omega} \leq c_{j}\left\|\psi_{j} v\right\|_{s, p, \Omega \cap \Omega},
$$

where $c_{j}$ does not depend on $v$. Therefore

$$
\begin{gathered}
\|v\|_{s, p, \Omega} \leq \sum_{j}\left\|\psi_{j} v\right\|_{s, p, \Omega} \leq \sum_{j} c_{j}\left\|\psi_{j} v\right\|_{s, p, \Omega \cap \Omega_{j}^{\prime}} \leq \\
\leq c \sum_{j}\left\|\psi_{j} v\right\|_{s_{, p, p} \Omega \cap \Omega_{j}} \leq c^{\prime} \sum_{j}\|v\|_{s, m, \Omega \cap \Omega_{j}^{\prime}}
\end{gathered}
$$

Since every term of the last sum is less than one of the addends of [2.13], the proof is complete.

Note that regularity assumptions such as [2.12] cannot be completely dropped. Actually, consider the open sets

$$
\Omega_{\mathrm{l}}=\left\{(\rho \cos \theta, \rho \sin \theta): 0<\rho<1,-\pi<\theta<\frac{\pi}{2}\right\}
$$




$$
\begin{gathered}
\Omega_{2}=\left\{(\rho \cos \theta, \rho \sin \theta): 0<\rho<1,-\frac{\pi}{2}<\theta<\pi\right\}, \\
\Omega=\Omega_{1} \cup \Omega_{2}
\end{gathered}
$$

and the function $v$ defined by

$$
v(\rho \cos \theta, \rho \sin \theta)=\theta, \text { for } 0<\rho<1,-\pi<\theta<\pi:
$$

it is $v_{\mid n_{i}} \in \mathbf{C}^{0.1}\left(\bar{\Omega}_{i}\right)$ for $i=1,2$, while $v \in \mathbf{W}^{s, p}(\Omega)$ if and only if $s<\frac{1}{p}$, because $|v|_{s, p, \Omega}=|v|_{s, p, B_{1}}$ for $0<s<1$.

The main step consists in obtaining an inequality analogous to [2.13], calling into play only seminorms. To this end, we state a known result (based on the compactness of the embedding of $\mathbf{W}^{s, p}(\Omega)$ in $\mathbf{L}^{\prime \prime}(\Omega)$, obtained by a combination of known results, e.g. [N] Theor. 6.3, [A] Theor. 7.48, [LM](iii) Prop. 2.7 and [LP] ch. V) as a lemma.

Lemma 2.6 Let $\Omega$ be a bounded, connected open subset of $\mathbf{R}^{N}$, of class $\mathrm{C}^{0.1}$ and $\omega \subset \Omega$ a nonempty open set; let $\left.s>0, p \in\right] 1,+\infty[$. Then there exists a positive constant $\mathrm{c}$ such that, for all $v \in \mathrm{W}^{s . p}(\Omega)$,

$$
\begin{gathered}
\|v\|_{s, p, \Omega} \leq c\left(|v|_{s, p, \Omega}+\|v\|_{s, p, \omega}\right) \\
\|v\|_{s, p, \Omega} \leq c\left(|v|_{s, p, \Omega}+\left|v_{\omega}\right|\right)
\end{gathered}
$$

where we have set

$$
v_{\omega}=\frac{1}{|\omega|} \int_{\omega} v
$$

Now we are in a position to prove the main result of this section.

Theorem 2.7 Let $\Omega$ and $\Omega_{i}(i=\mathrm{I}, \ldots, n)$ be bounded, connected open subsets of $\mathbf{R}^{N}$ verifying condition [2.12]. Assume that $\Omega_{i}$ is of class $\mathbf{C}^{0.1}$ and that $\Omega=\bigcup_{i} \Omega_{i:}$ If $\left.\left.s \in\right] 0,1\right]$ and $\left.p \in\right] 1,+\infty[$ then there is a constant $c>0$ such that for all $v \in \mathbf{W}^{s, p}(\Omega)$

$$
|v|_{s, p, \Omega} \leq c \sum_{i}|v|_{s, p, \Omega_{i}}
$$

Proof. In the following, $c$ will denote different constants, independent of v. Let us consider [2.13]: thanks to Lemma 2.3 we may suppose that each of 
the $\Omega_{i}$ 's, except the first, intersects one of the preceding ones, which we will denote by $\Omega_{i}^{\prime}$. Then, for $\mathrm{I}<i \leq n$ it is

$$
\|v\|_{s, p, \Omega_{i}} \leq c\left(|v|_{s, p, \Omega_{i}}+\|v\|_{s, p, \Omega_{i}^{\prime} \cap \Omega_{i}}\right) \leq c\left(|v|_{s, p, \Omega_{i}}+\|v\|_{s, p, \Omega_{i}^{\prime}}\right),
$$

where [2.14] has been used. Iterating this inequality, adding up and applying [2.15] we find

$$
\|v\|_{s, p, \Omega} \leq c\left(\sum_{i}|v|_{s, p, \Omega_{i}}+\|v\|_{s, p, \Omega_{1}}\right) \leq c\left(\sum_{i}|v|_{s_{1}, n_{1} n_{i}}+\left|v_{\Omega_{1}}\right|\right),
$$

whence

$$
|v|_{s, p, \Omega} \leq c\left(\sum_{i}|v|_{s, p, \Omega_{i}}+\left|v_{\Omega_{1}}\right|\right)
$$

Applying [2.18] to $v-v_{\Omega_{1}}$ we get [2.17].

We end this section with a result which will be used in the next one.

Lemma 2.8 Let $\Omega$ and $\Omega^{\prime}$ be open subsets of $\mathbf{R}^{N}$. Let $\alpha: \Omega \rightarrow \Omega^{\prime}$ be a diffeomorphism of class $\mathbf{C}^{m, 1}$. If $\left.\left.\left.s \in\right] 0, m+1\right], p \in\right] 1,+\infty\left[\right.$ and $v \in \mathbf{W}^{s, p}\left(\Omega^{\prime}\right)$ then $v_{\circ} \alpha \in \mathbf{W}^{s, p}(\Omega)$ and

$$
\left|v_{o} \alpha\right|_{s, p, \Omega} \leq c|v|_{s, p, \Omega^{\prime}} \text { and }\left.\left\|v_{o} \alpha\right\|\right|_{s, p_{s} \Omega} \leq c\|v\|_{s, p, \Omega l^{\prime}},
$$

where $\mathrm{c}$ does not depend on $v$.

Proof. The second inequality is well known (see [LMI] 12.9, which extends to our case). Yet, its "natural" proof shows also the first one: indeed, if $s>1$, lower order derivatives of $v$ do appear in evaluating higher derivatives of $v_{0} \alpha$, but $v$ itself does not; now, this is exactly what is needed to express . the seminorm according to the definitions [2.6] and [2.9]. If $s \leq 1,[2.19]$ is immediate.

\section{THE CASE OF A MANIFOLD}

Now we are able to deal with a manifold $\Gamma$ of a euclidean space. The definition of $\mathbf{W}^{x, p}(\Gamma)$ is clearly equivalent to the usual one, given by means of local charts and partition of unity.

Definition 3.1 Assume [1.l] and let $0<s \leq m+1$ and $1<p<\infty$. We say that a function $v$ of $\mathrm{L}^{p}(\Gamma)$ belongs to $\mathbf{W}^{s, p}(\Gamma)$ when for all $x \in \Gamma$ there exists a diffeomorphism $\alpha: B_{1} \rightarrow \alpha\left(B_{1}\right) \subset \Gamma$ of class $C^{m .1}$ such that $x \in \alpha\left(B_{1}\right)$ and $v \circ \alpha \in \mathbf{W}^{s, p}\left(B_{1}\right)$. 
In defining the norm in $\mathbf{W}^{s, r}(\Gamma)$ we will not employ partition of unity, as usually done; the method allows us to define a seminorm as well.

Definition 3.2 Under the assumptions of Definition 3.1, let $\left\{\alpha_{i}\right\}$ be a finite family of diffeomorphisms $\alpha_{i}: B_{1} \rightarrow \alpha_{i}\left(B_{v}\right) \subset \Gamma$ of class $C^{m, 1}$ such that $\left\{\alpha_{i}\left(B_{1 / 2}\right)\right\}$ is an open covering of $\Gamma$. For $v \in \mathbf{W}^{s, p}(\Gamma)$ we set:

$$
\begin{aligned}
\|v\|_{s, p, \Gamma} & =\sum_{i}\left\|v_{0} \alpha_{i}\right\|_{s, p, B_{1 / 2^{*}}} \\
\|\left. v\right|_{s, p, \Gamma} & =\sum_{i}\left|v_{0} \alpha_{i}\right|_{s, p, B_{1 / 2}}
\end{aligned}
$$

Obviously [3.1] and [3.2] depend on the choice of the system $\left\{\alpha_{i}\right\}$; yet, different systems lead to topologically equivalent results, thanks to the following Theorem.

Theorem 3.3 Assume [I.I] and let $0<s \leqslant m+1$ and $1<p<\infty$. Let $\left\{\alpha_{i}\right\}$ and $\left\{\beta_{j}\right\}$ be two families satisfying the assumptions of Definition 3.2. Then there exists $c>0$ such that for all $v \in \mathbf{W}^{s, p}(\Gamma)$ it is:

$$
\begin{aligned}
\sum_{i}\left|v_{0} \alpha_{i}\right|_{s, p, \beta_{1 / 2}} & \leq c \sum_{j}\left|v_{\circ} \beta_{j}\right|_{s, p, B_{1 / 2}}, \\
\sum_{i}\left\|v_{\diamond} \alpha_{i}\right\|_{s, p, \beta_{1 / 2}} & \leq c \sum_{j}\left\|v_{\circ} \beta_{j}\right\|_{s, p, B_{1 / 2}} .
\end{aligned}
$$

Proof. It is enough to evaluate each of the terms on the left hand side of [3.3] or [3.4] by means of the corresponding right hand side. For this, fix $i$ and set $\alpha=\alpha_{i}$ and $B=B_{1 / 2}$. We would like to use Lemma 2.5 and Theorem 2.7 with $\Omega=B$ and $\Omega_{j}=\alpha^{-1}\left(\beta_{j}(B)\right)$. However, these $\Omega_{j}$ 's may be irregular, hence we introduce some auxiliary open sets as follows.

For all $x \in \bar{B}$ fix $j=j(x)$ and $\epsilon=\epsilon(x)$ such that $B_{\mathrm{e}}(x) \subset B_{1}$ and $\alpha\left(B_{\epsilon}(x)\right) \subset \beta_{j}(B)$; using the compactness of $\bar{B}$, we may find a finite number of points $x^{k} \in \bar{B}$ such that, setting $\tilde{\Omega}_{k}=B_{\left.\epsilon x^{k}\right)}\left(x^{k}\right)$, it is: $\bar{B} \subset \cup \widetilde{\Omega}_{k} \subset B_{1}$, $\alpha\left(\widetilde{\Omega}_{k}\right) \subset \beta_{j_{k}}(B)$, for suitable $j_{k}$. Set $\Omega_{k}=B \cap \tilde{\Omega}_{k}$ and $\Omega_{k}^{\prime}=\left(\beta_{j_{k}}^{-1} \circ \alpha\right)\left(\Omega_{k}\right)$ and denote by $c$ different constants independent of $v$. We shall show that the inequality

$$
\left|v_{0} \alpha\right|_{s, p, B} \leq c \sum_{k}\left|v_{0} \beta_{j k}\right|_{s, p, \Omega_{k}}
$$

and the analogous for the norms hold, distinguishing the cases $0<s \leq 1$ and $s>1$. 
If $0<s \leq 1$, Theorem 2.7 and Lemma 2.8 give:

$$
\begin{aligned}
\left|v_{0} \cdot \alpha\right|_{s, p, B} & \leq c \sum_{k}\left|v_{0} \alpha\right|_{s, p, \Omega_{k}}=c \sum_{k}\left|\left(v_{\circ} \beta_{j k}\right) \circ\left(\beta_{j_{k}}^{-1} \circ \alpha\right)\right|_{s, p_{r} \Omega_{k}} \leq \\
& \leq c \sum_{k}\left|v_{0} \beta_{j_{k}}\right|_{s, p, \Omega_{k}^{\prime}}
\end{aligned}
$$

and [3.3] follows.

Let $s>1$. Denoting by $\gamma^{l}$ the $l$-th component of the generic function $\gamma$ with values in $\mathbf{R}^{N}$, we have:

$$
\begin{aligned}
\left|v_{0} \alpha\right|_{s, p, B} & =\sum_{h}\left\|\partial_{h}\left(v_{0} \alpha\right)\right\|_{s-1, p, B} \leq c \sum_{h} \sum_{k}\left\|\partial_{h}\left(v_{\circ} \alpha\right)\right\|_{s-1, p, \Omega_{k}}= \\
& =c \sum_{h} \sum_{k}\left\|\partial_{h}\left[\left(v_{0} \beta_{j_{k}}\right) \circ\left(\beta_{j_{k}}^{-1} \circ \alpha\right)\right]\right\|_{s-1, p_{,} \Omega_{k}}= \\
& =c \sum_{h} \sum_{k}\left\|\sum_{l}\left\{\left[\partial_{l}\left(v_{0} \beta_{j_{k}}\right)\right] \circ\left(\beta_{j_{k}}^{-1} \circ \alpha\right)\right\} \partial_{h}\left(\beta_{j_{k}}^{-1} \circ \alpha\right)^{l}\right\|_{s-1, p_{1} \Omega_{k}} \leq \\
& \leq c \sum_{k} \sum_{l}\left\|\left[\partial_{l}\left(v_{0} \beta_{j_{k}}\right)\right] \circ\left(\beta_{j_{k}}^{-1} \circ \alpha\right)\right\|_{s-1, p_{1}, \Omega_{k}} \leq \\
& \leq c \sum_{k} \sum_{l}\left\|\partial_{l}\left(v_{0} \beta_{j_{k}}\right)\right\|_{s-1, p, \Omega_{k}^{\prime}}=c \sum_{k}\left|v_{0} \beta_{j_{k}}\right|_{s, p, \Omega^{\prime}}
\end{aligned}
$$

and [3.3] still follows.

The analogous inequalities for the norms are proved in the same way.

\section{FURTHER REMARKS}

Our aim is firstly comparing the norm [3.1] with the usual one, whose definition exploits a partition of unity.

Let $\left\{\alpha_{i}\right\}$ be like in Definition 3.2 and let $\left\{\psi_{i}\right\}$ be a partition of unity of class $C^{m, 1}$ associated to the compact $\Gamma$ and to the covering $\left\{\alpha_{i}\left(B_{1 / 2}\right)\right\}$. Define (see. [LM](iii) 2.5)

$$
\|v\|_{s, p, \Gamma}^{*}=\sum_{i}\left\|\left(v \psi_{i}\right)_{\circ} \alpha_{i}\right\|_{s, p, B_{12}} .
$$

Proposition 4.1 Assume [I.I] and let $0<s \leq m+1$ and $1<p<\infty$. The norms [3.I] and [4.I] are equivalent. 
Proof. Using Lemma 2.4, we have immediately:

$$
\|v\|_{s, p, \Gamma^{\prime}}^{*}=\sum_{i}\left\|\left(v_{0} \alpha_{i}\right)\left(\psi_{i} \circ \alpha_{i}\right)\right\|_{s, p, B_{1,2}} \leq c \sum_{i}\left\|v_{0} \alpha_{i}\right\|_{s, p, B_{1,2}}=c\|v\|_{s, p, l},
$$

where $c$ does not depend on $v$.

Since $\mathbf{W}^{s, p}(\Gamma)$ is complete with respect to [4.1], the assertion will follow from Banach theorem as soon as we show that $\mathbf{W}^{s, p}(\Gamma)$ is complete with respect to [3.1]. Let $\left\{v_{n}\right\}$ be a Cauchy sequence with respect to [3.1]: then, for all $i,\left\{v_{n}\right.$ o $\left.\alpha_{i}\right\}$ converges in $W^{s, p}\left(B_{t / 2}\right)$ to a function $w_{i}$. We only have to prove the existence of a function $v \in L^{p}(\Gamma)$ such that $v_{0} \alpha_{i}=w_{i}$ for all $i$. Since the set of indices is finite, there is a subsequence $\left\{v_{n_{k}}\right\}$ such that $v_{n_{k}} \alpha \alpha_{i} \rightarrow w_{i}$ a.e. in $B_{1 / 2}$; it follows that $v_{m_{k}} \rightarrow w_{i} \circ \alpha_{i}^{-1}$ a.e. in $\alpha_{i}\left(B_{1 / 2}\right)$, whence $w_{i} \circ \alpha_{i}^{-1}=w_{j} \circ \alpha_{j}^{-1}$ in $\alpha_{j}\left(B_{1 / 2}\right) \cap \alpha_{j}\left(B_{1 / 2}\right)$ and $v$ is defined in an obvious manner.

Let us introduce other possible norms in $\mathbf{W}^{s, p}(\Gamma)$, related to the seminorm [3.2], equivalent to [3.1].

Proposition 4.2 Assume [I.I] and let $0<s \leq m+1$ and $1<p<\infty$. Suppose $\Gamma$ is connected and let $\omega \subset \Gamma$ be a set with positive $\mathrm{N}$-dimensional measure. Then the following norms are equivalent to [3.1]:

$$
\begin{gathered}
\|v\|_{s, p, J^{\prime}}^{\prime}=|v|_{s, p, \Gamma^{+}}+\|v\|_{0, p, \omega}, \\
\|v\|_{s, p, \Gamma^{\prime}}=|v|_{s, p, \Gamma}+\left|v_{\omega}\right|,
\end{gathered}
$$

where $\left\|_{\bullet}\right\|_{0, p, \omega}$ is the norm in $\mathrm{L}^{\prime}(\omega)$ and $v_{\omega}$ is the average of $v$ on $\omega$.

Proof. Obviously [4.2] and [4.3] are estimated from above by [3.1]. Converse inequalities are of Friedrichs-Poincaré type and can be obtained according to classical arguments, provided one notices that:

(i) $|v|_{s, p, \Gamma}=0$ is equivalent to $v=$ constant, so that [4.2] and [4.3] are norms;

(ii) the embedding of $\mathbf{W}^{n, p}(\Gamma)$ in $L^{p}(\Gamma)$ is compact with respect to the norm $[4,1]$, hence with respect to [3.1], too.

Proposition 4.3 Assume [I.I] and let $0<s \leq m+1$ and $1<p<\infty$. Suppose $\Gamma$ is connected and set

$$
|v|_{s, p, \Gamma^{\prime}}^{*}=\inf \left\{\|v+k\|_{s, p, \Gamma}: k \in \mathbf{R}\right\} .
$$


Then [4.4] and [3.2] are equivalent.

Proof. For all $k \in \mathbf{R}$ one has

$$
|v|_{s, p, \Gamma}=|v+k|_{s, p, \Gamma} \leq\|v+k\|_{s, p, \Gamma}
$$

whence $|\nu|_{s, p, \Gamma} \leq|v|_{s, p, \Gamma}^{*}$. Denoting by $v_{\Gamma}$ the average of $v$ on $\Gamma$, thanks to the previous Proposition one has

$$
|v|_{s, p, \Gamma}^{\bullet} \leq\left\|v-v_{\Gamma}\right\|_{s, p, \Gamma} \leq c\left|v-v_{\Gamma}\right|_{s, p, \Gamma}=c|v|_{s, p, \Gamma} .
$$

If $\Gamma$ is also the boundary of an open set, then there is a further possibility.

Proposition 4.4 Let $\Omega \subset \mathbf{R}^{N+1}$ be a bounded, connected open set of class $\mathrm{C}^{m, 1}$ and let $\Gamma$ be its boundary, which is assumed to be connected; let $0<s \leq m+1$ and $1<p<\infty$. Suppose either $p=2$ or $\mathrm{s}$ not integer.

For $v \in W^{s . p}(\Gamma)$, define $\tilde{v}$ by means of: $\Delta \tilde{v}=0$ in $\Omega$ and $\tilde{v}=v$ on $\Gamma$. Introduce

$$
|v|_{s, p, \mathrm{\Gamma}}^{*}=|\tilde{v}|_{s}+\frac{1}{p}, p, \mathrm{n} \mathbf{1} .
$$

Then [4.5] and [3.2] are equivalent.

Proof. We shall use well known results ([LM](v), Theorem 4.2, [LM] (vi) Theorem 4.1). For all $k \in \mathbf{R}$ it is

$$
\begin{gathered}
|v|_{s, p, 1}^{*}=|\tilde{v}|_{s+\frac{1}{p}, p, \Omega}=|\tilde{v}+k|_{s+\frac{1}{p}, p, \Omega}=\left|(v+k)^{\sim}\right|_{s+\frac{1}{p}, p, \Omega} \leq \\
\leq\left\|(v+k)^{\sim}\right\|_{s+\frac{1}{p}, p, \Omega} \leq c\|v+k\|_{s, p, \Gamma} .
\end{gathered}
$$

By the previous Proposition we then find

$$
|v|_{s, p, \Gamma}^{*} \leq c|v|_{s, p, \mathrm{I}}^{*} \leq c|v|_{s, p, \Gamma} .
$$

Moreover, choosing $k$ to be the average of $\tilde{v}$ in $\Omega$ and applying [2.15], we obtain:

$$
\begin{gathered}
\quad|v|_{s, p, \Gamma}=|v-k|_{s, p, \Gamma} \leq\|v-k\|_{s, p, \Gamma} \leq c\left\|(v-k)^{\sim}\right\|_{s+\frac{1}{p}, p, \Omega}= \\
=c \| \tilde{v}-\left.k\right|_{s+\frac{1}{p}, p, \Omega} \leq c|\tilde{v}-k|_{s+\frac{1}{p} \cdot p, \Omega}=c|\tilde{v}|_{s+\frac{1}{p}, p, \Omega}=c|v|_{s, p, \Gamma}^{*} .
\end{gathered}
$$




\section{References}

[A] ADAms, R. Sobolev spaces. Academic Press. New York (1975).

[BL] BERGH, J. and LOFSTROM, J. Interpolation spaces: an introduction. SpringerVerlag. Berlin (1976).

[GG] GASTAI.DI, F. and GILARDI, G. A class of noncoercive variational inequalities. (To appear).

[KJF] Kufner, A., John, O. and Fucik, S. Funcion spaces Academia, PrahaNoordhoff, L.eyden (1977).

[LM]' LIONS, J. L. and MAGEnes, E. Problèmes aux limites non homogènes. (iii) Ann. Scuola Norm. Sup. Pisa cl. Sci., IlI, 15 (1961), 39-101; (v) Ann. Scuola Norm. Sup. Pisa cl. Sci., III, 16 (1962), 1-44; (vi) J. Analyse Math. 11 (1963), 165-188.

[LM1] LIONS, J. L. and MAGINES, E. Non-homogeneous boundary value problems and applications. Vol. 1, Springer-Verlag, Berlin (1972).

[LP] LIONS, J. L. and PEETRE, J. Sur une classe d'espaces dinterpolation. Inst. Hautes Etudes Sc. Publ. Math. 19 (1964), 5-68.

[M] MAGENES, E. Spazi di interpolazione ed equazioni a derivate parziali. Atti VII Congresso U.M.I., Genova 1963. Cremonese, Roma (1964), 134-197.

[N] NECAS, J. Les méthodes directes en théorie des équations elliptiques. Masson, Paris-Academia, Praha (1967). .

[T] TRIEBEL, H. Interpolation theory, function spaces, differential operators. North Holland, Amsterdam (1978).

Dipartimento di Matematica dell'Università

Strada Nuova, 65, 27100 Pavia.

Italia. 